\title{
Stochastic simulation of maize productivity: spatial and temporal uncertainty in order to manage crop risks
}

\author{
A. R. L. Grifo ${ }^{1,2}$ J. R. Marques da Silva ${ }^{2,3}$
}

Published online: 24 May 2015

(C) Springer Science+Business Media New York 2015

\begin{abstract}
There is emerging interest in evaluating the uncertainty of agricultural production to support the production process and for guidance in decision making. The main objective of this work was to estimate the spatial and temporal maize yield uncertainty using stochastic simulation techniques to reduce the economic risk considering the producer risk profile and the international prices of maize and inputs. The results showed that (i) the class yield percentage variation in yield stochastic simulation depends on the sampling density; (ii) higher sampling densities promote an overestimation of low and high yield values compared to those of real yield data; (iii) reducing sampling density promotes the low and high values of overestimation reduction while increasing the central classes values compared to those of real yield data; (iv) the ideal point density for yield stochastic simulation is approximately 65 points/ha; (v) in Mediterranean environments, more than 3-4 years' worth of real yield data considered as a whole do not seem to improve the parcel level of confidence when cropping irrigated maize; and (vi) the number of equiprobable surfaces that were generated by sequential Gaussian simulation helped to calculate the yield class uncertainty and permitted the study of class yield probabilities for a particular position of the parcel and, therefore, to manage the yield risk and support future decisions. The approach that is presented in this paper may increase prior knowledge of
\end{abstract}

Electronic supplementary material The online version of this article (doi:10.1007/s11119-015-9401-1) contains supplementary material, which is available to authorized users.

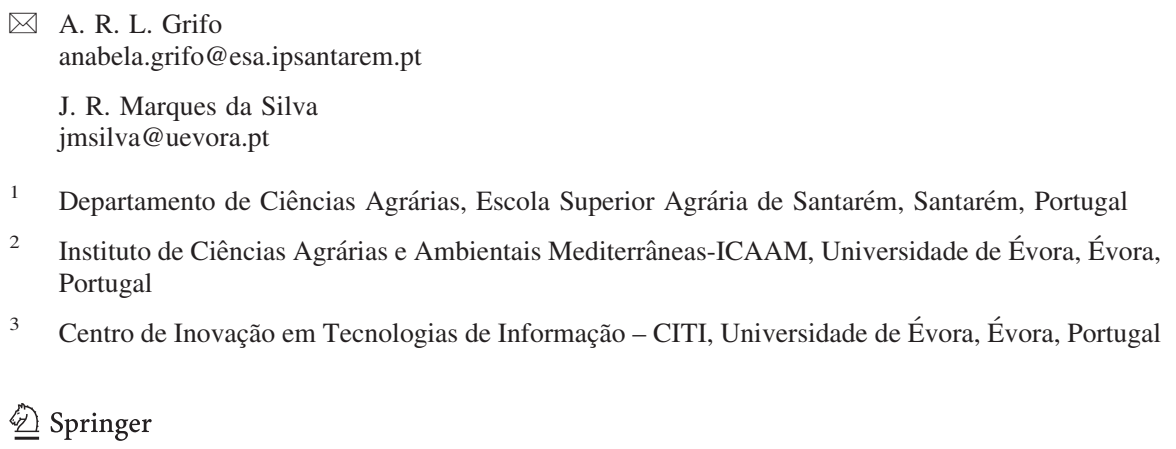

